\title{
Evolución de la voz desde el nacimiento hasta la senectud
}

* Clara Aponte G.
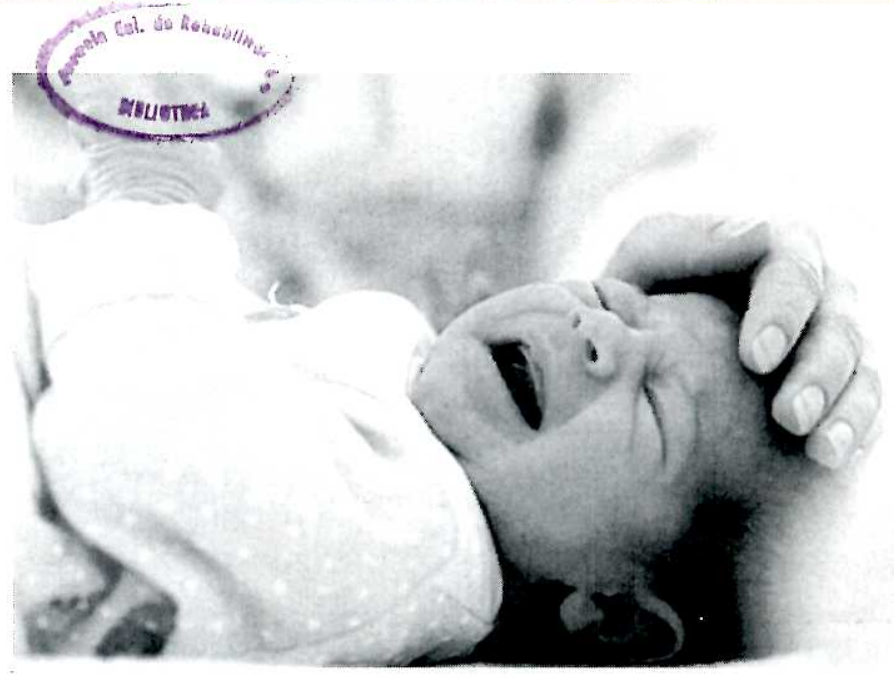

\section{RESUMEN}

En este artículo, se hace una revisión sobre los cambios que sufre la voz en el decurso de la vida, llevando a cabo un recorrido, desde la etapa infantil, pasando por la pubertad y adolescencia, la etapa presenil, para finalizar en la etapa senil. Por último, se concluye, en la importancia de la preservación de la salud vocal, como una manera de brindar calidad de vida a los individuos, siendo esta última, una meta de la rehabilitación.

PALABRAS CLAVE: Voz, fonación, calidad de la voz, cuerdas vocales.

\section{NTRODUCCIÓN}

En el decurso de la vida la voz sufre diferentes cambios que obedecen a factores de desarrollo, donde intervienen de manera preponderante, el sistema nervioso y el sistema hormonal.

La voz infantil, asexual y aguda, y la voz adulta que se instaura hacia los 18 años, permanece con todas sus características hasta la época de la menopausia en la mujer y la andropausia en el hombre, apareciendo en esta etapa alteraciones que pueden ser marcadas o no, para desembocar finalmente en la cuarta edad, en la que se suceden modificaciones importantes, evidenciándose un franco deterioro. De esta forma se puede afirmar que la voz refleja el comportamiento anátomo fisiológico del hombre a lo largo de la vida. Para efectos del presente artículo, se tomará el ciclo vital del hombre dividido en cinco edades, analizando en cada una de ellas, las características vocales correspondientes:

INFANTIL: Comprende desde el nacimiento hasta los 9 años.

PUBERTAD Y ADOLESCENCIA: 10 a 17 años.
ADULTEZ - MADUREZ: 18 a 49 años.

PRESENIL O CLIMATERIO: 50 a 77 años.

SENIL: 77 años en adelante.

\section{ETAPA INFANTIL (O A 9 ANOS)}

La función vocal de la laringe de un niño comienza desde el nacimiento. La modulación y entonación de los sonidos que produce, tienen una información psicoafectiva, que es muy bien interpretada por la madre.

Según, J. Verhulst (1987), en el momento del nacimiento, la laringe tiene un tamaño aproximado de un tercio de la laringe de una mujer adulta, los pliegues vocales

* Fonoaudióloga egresada de la Escuela Colombiana de Rehabilitation y Especialista en Docencia Universitaria. Docente de las áreas de lenguaje, voz y habla de la Escuela Colombiana de Rehabilitación. 
miden aproximadamente 4.5 a 5 milímetros, estando constituidos, la mitad por las apófisis vocales de los aritenoides y la otra mitad por la porción músculomembranosa. Heuillet (1997), afirma que en el nacimiento, el hioides está situado en el tercio inferior de la segunda vertebra cervical (C2). El cartílago cricoides en su borde inferior, se encuentra ubicado entre C3 y C4; el plano gótico está hacia el medio de la tercera vértebra cervical (C3).

Esta posición alta de la laringe, con el borde libre de la epíglotis que se sitúa muy próxima del velo del paladar, hace que el modo respiratorio sea nasal casi obligatorio, hasta la edad de cuatro meses.

En el recién nacido la musculatura laríngea está constituida por fibras tipo 2 que son de contracción rápida y corta, tienen un rol esfinteriano de cierre rápido para protección durante la alimentación. Las fibras de tipo I de contracción lenta y prolongada, inicialmente no son numerosas, poco a poco van en aumento, permitiendo la modulación de la voz y el sostenimiento prölongado del sonido necesario para la voz hablada y en mayor proporción para la voz cantada.

Según Sedlakoba y Aronson, citados por Dalleas (1987), el primer grito tiene una tonalidad media entre 440 y $500 \mathrm{~Hz}$, que se corresponden en la escala musical con la nota La 3, con una intensidad entre los 80 y $90 \mathrm{~dB}$; el timbre es pobre en armónicos, con un predominio en las frecuencias agudas. Según Heuillet (1997), el primer formante se sitúa entre 2000 y $3000 \mathrm{~Hz}$.

A los dos meses de vida se adquiere ya una tesitura o extensión vocal de cinco semitonos, con un uso privilegiado de figuras melódicas, tales como, los glisados hacia el agudo.

De los tres a los nueve meses, el niño juega con su aparato vocal y hacia el final de esta edad comienza a imitar y a reproducir ritmos y melodías. A los siete meses, la tesitura alcanza un poco menos de una octava, hay más riqueza en cambios tonales, se pueden producir sonidos fuertes y prolongados. El descenso de la laringe, que ocurre entre el nacimiento y la edad de dos años, permite una ganancia en tonos graves. El cartílago cricoides, que al nacimiento estaba a la altura de C3, a los dos años desciende a C5.

A esta edad, es muy importante el desarrollo del lenguaje, esto impone a la voz, variaciones en el tono y la frecuencia.

Hacia los tres años la frecuencia fundamental se estabiliza alrededor de los $318 \mathrm{~Hz}$. En esta edad el niño utiliza el grito, hace esfuerzo vocal y su voz de niño está influenciada por su temperamento; una voz débil muestra un niño tímido y una voz fuerte muestra a un niño despierto e inquieto.

La constitución de la voz, depende ciertamente de la anatomía de la laringe y de los resonadores pero, el medio no solamente linguístico sino vocal tiene una importancia fundamental. Tambien existen otros factores que vale la pena mencionar como son: hereditarios, genéticos, de temperamento y personalidad.

Entre los cuatro y los seis años la extensión es inferior a una octava. Según Dalleas,(1987), el tono fundamental a los siete años desciende, situándose alrededor de los $250-280 \mathrm{~Hz}$. ( Si2- Do3 de la escala musical). Según Woisard et Al. (1996), a los siete años la frecuencia fundamental, es de $295 \mathrm{~Hz}$ para las niñas y $268 \mathrm{~Hz}$. para los niños, estableciéndose así ya una diferencia entre la voz de la niña y la del niño. Hacia los ocho años, la voz cantada se extiende a dos octavas aproximadamente.

Con el crecimiento y el dominio progresivo del soplo, se produce una ganancia en intensidad y en estabilidad de la producción sonora, que va a beneficiar la voz cantada.

\section{ETAPA DE LA PUBERTAD Y ADOLESCENCIA (10 A 17 AÑOS)}

En esta etapa, la voz, especialmente la masculina, sufre una serie de cambios importantes. El adolescente abandona el registro agudo para optar por la voz del adulto. Este pasaje se efectúa habitualmente por una adaptación morfológica de la laringe, al desarrollo del organismo.

En el varón se presenta un rápido crecimiento de todos los cartílagos laríngeos, sobretodo de los aritenoides, con el consiguiente aumento del tamaño de la laringe, en todos los diámetros, prevaleciendo el anteroposterior. Según Sataloff (1991), el ángulo entre las dos láminas tiroideas se hace más agudo, aproximadamente 90 grados, la laringe desciende. Verhulst (1987), ubica los pliegues vocales en el borde inferior de la quinta vertebra cervical (C5). Los músculos intrínsecos, tambien son obligados a una adaptación, que se traduce, en un aumento de la longitud y espesor de los pliegues vocales verdaderos, según Woisard (1996), los pliegues vocales pasan de 17 a 28 milímetros de longitud. Estos 
cambios anatómicos producen un descenso de la frecuencia fundamental de una octava, aproximadamente $110 \mathrm{~Hz}$., pasando del registro de pecho al registro de cabeza, la laringe debe entonces, responder a estimulaciones monofásicas. En el comienzo de la muda (cambio de voz), la voz cantada se hace casi imposible, la extensión vocal crece rápidamente hacia los tonos graves y la intensidad aumenta.

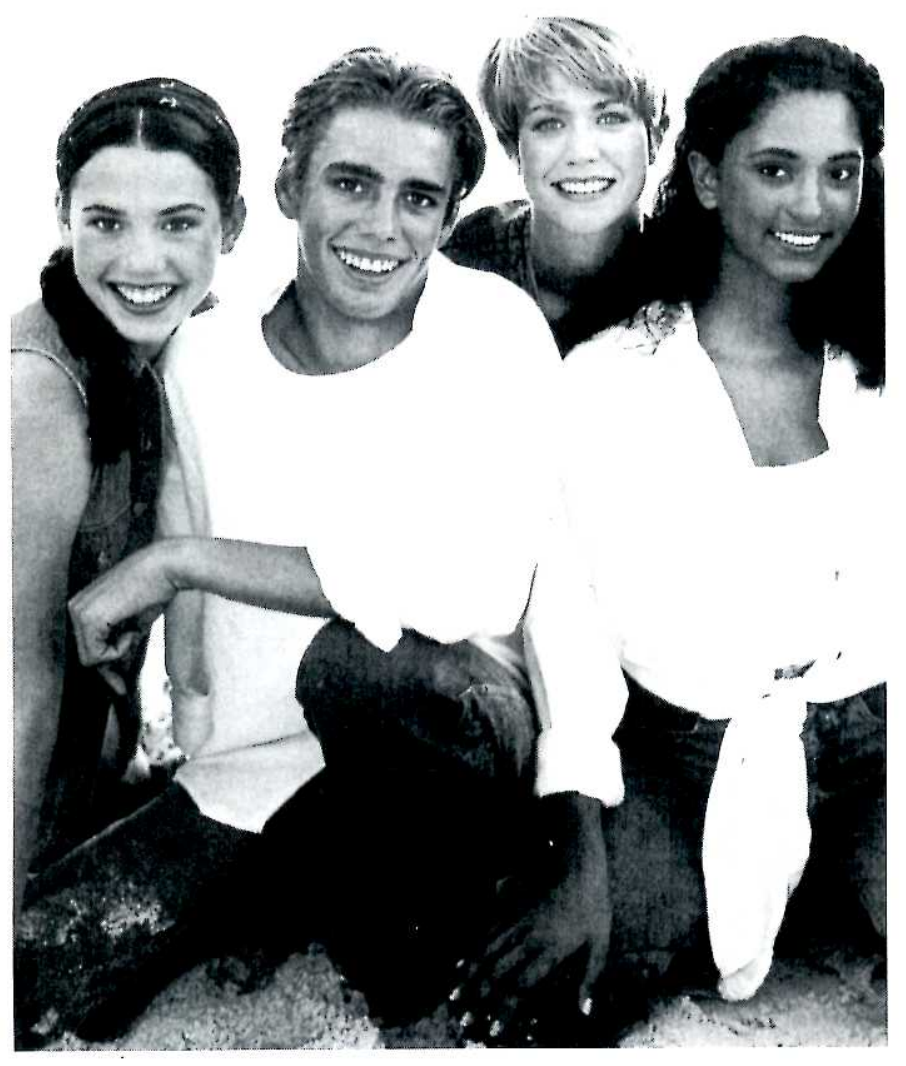

Esta intensa actividad muscular y cartilaginosa en el hombre, se traduce en una hiperemia marcada de la mucosa laríngea, especialmente de los aritenoides y de las bandas ventriculares. El desarrollo pulmonar y de las cavidades de resonancia del aparato fonatorio, condicionan el cambio de la voz, iniciándose éste, según Woisard et Al., (1996), alrededor de los 13 años, pero puede estar tambien, entre los 10.5 años y los 18.5 años con una duración entre 8 y 26 meses.

Igualmente la muda se acompaña de cambios hormonales, que conllevan manifestaciones afectivas, psíquicas y sociales. La identificación de la voz del padre en oposición a la de la madre, el éxito en las diferentes etapas de maduración de la personalidad, va ha condicionar el paso de la voz infantil, a la voz del adulto.
Por consiguiente, si hay algún trastorno de la muda, esta dificultad de adaptación del comportamiento fonatorio, a una dimención adulta de la laringe, debe ser considerada como un retardo de maduración psicológica, y más específicamente en el hombre es como una resistencia a la aceptación de su nueva identificación sexual. Esta mutación, puede manifestarse de manera patológica, presentándose bitonalidad, diplofonía y voz de falsete mutacional.

La muda existe tambien en la mujer, aunque aquí es menos evidente la modificación del registro. La altura promedio, baja una tercera, siendo este cambio poco perceptible, igualmente las modificaciones del timbre. El proceso evolutivo anatómico aparece en menor grado y el aumento de la laringe se efectúa, más en el diámetro vertical. El ángulo del cartílago tiroides es de 120 grados y según Sataloff (1991), la frecuencia fundamental está alrededor de los $220-225 \mathrm{~Hz}$. Según Woisard (1996), los pliegues vocales, aumentan su longitud de 17 a 23 milímetros, la extensión vocal, al igual que en el hombre, está alrededor de tres octavas.

Algunas mujeres conservan su voz de niñas aún en la edad adulta, generalmente lo hacen con el fin de obtener ganancias con el sexo opuesto, para dar la impresión de debilidad, o también por factores estéticos. En estos casos, la laringe es normal, pero se sitúa en una posición alta, que facilita el tono agudo.

\section{ETAPA DE LA MADUREZ O ADULTEZ (18 A 49 AÑOS)}

Las características de la voz, obtenidas en el periodo puberal y la adolescencia, se desarrollan hasta llegar a los caracteres estables de la edad adulta. Para lograr el mantenimiento de esta voz, tiene mucha importancia el funcionamiento de las hormonas sexuales.

Según Sataloff (1991), la laringe desciende a la altura de la séptima vértebra cervical (C7), entre los 15 y 20 años, así la mujer tiene su tono fundamental alrededor de los $225 \mathrm{~Hz}$. Las cuerdas vocales, a medida en que se avanza de edad, van perdiendo elasticidad y fibras de colágeno. Estos factores intervienen, sobretodo en la voz cantada, especialmente en la mujer, donde la queja principal es la pérdida de agudos, tendencia a bajar el tono y opacamiento del timbre.

En el hombre la voz tiende a conservarse mejor que en la mujer. 


\section{ETAPA PRESENIL O CLIMATERIO (49 A 77 AÑOS)}

En esta época, aparecen en los dos sexos, profundos cambios en el equilibrio hormonal, aunque en el hombre por una reducción gradual de andrógenos, se modifica muy poco la voz.

Según E. Pícoli (1984), en el proceso de involución laringofónico, se pueden describir tres periodos con características propias:

1. Periodo premenopáusico.

2. Periodo menopáusico.

3. Periodo post-menopáusico o gerofónico propiamente dicho.

En el presente artículo se partirá del segundo periodo, teniendo en cuenta que es el correspondiente a la etapa tratada.

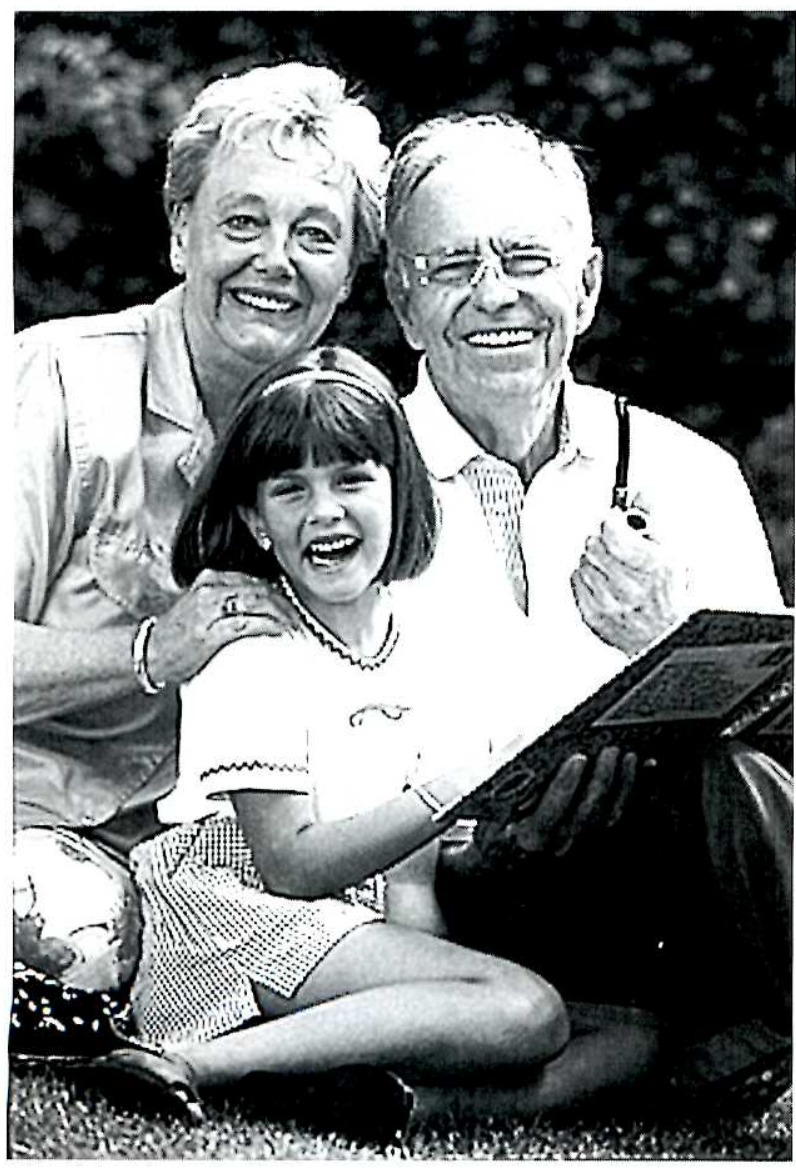

Durante el Periodo menopáusico (45 a 60 años), en la mujer, hay un descenso en la producción de estrógenos, por lo cual la laringe se convierte en una laringe menopáusica, cumpliéndose lo que se podría llamar la segunda mutación y en algunos casos, ésta puede llegar al virilismo; se pueden observar pliegues vocales edematosos y fonación de bandas, pérdida de agudos, aumento de la tesitura hacia los graves, timbre engrosado y resonancia de predominio pectoral. En el hombre hay reducción de andrógenos y se encuentra con frecuencia, presencia de edemás, pólipos y fonación de bandas ventriculares.

En cantantes y actores, hay descenso del tono fundamental, disminución del tonismo glótico, inseguridad en el ataque vocal, disminución en la extensión vocal, uso incorrecto de la respiración y se compromete la voz media. Ante estos cambios, los profesionales de la voz hacen con frecuencia una serie de adaptaciones, produciendo malos hábitos vocales, como el carraspeo o posturas inadecuadas. En los cantantes se ve a menudo un cambio en la clasificación, otras veces, la voz permanece inalterable, esto se explica por factores de orden psíquico y físico. Pero aparte de las excepciones, luego de la madurez vocal, sigue un lento y progresivo decaimiento anátomo funcional de la laringe, que evolucionará hacia las alteraciones seniles.

\section{ETAPA SENIL (77 AÑOS EN ADELANTE)}

La voz depende de la concurrencia de muchos sistemas: neurológico, hormonal, auditivo, respiratorio, muscular, óseo, psíquico, entre otros. Las alteraciones en estos sistemas contribuyen al deterioro vocal.

En estudio practicado por Honjo, Iwao e Isshiki (1980), sobre la laringoscopia y características vocales en ancianos en edad de 75 años 20 hombres y 20 mujeres, se pudo observar que en los hombres existe tendencia a mostrar marcada atrofia cordal en un $67 \%$, y/o edema de los pliegues vocales en el $39 \%$, con un tono más alto que el de los hombres jóvenes.

En las mujeres se vio edema cordal y leve ronquera, con un tono más bajo que en las mujeres jóvenes, y atrofia cordal en un 26\%; en los hombres se vio atrofia en un $67 \%$; hiatus en el $67 \%$ de los hombres y en un $58 \%$ de las mujeres, sulcus vocalis, se presentó en el $10 \%$ de los hombres y las mujeres.

Con relación al sistema neurológico, la producción vocal necesita de una correcta acción muscular. Como es sabido, en el anciano, aparecen placas seniles, aumento de gliosis y disminución de las fibras nerviosas; 
las neuronas muestran atrofia y degeneración nuclear de las neurofibrillas, esto lleva a una acción lenta, presencia de temblor y disminución del vigor físico; este deterioro progresivo de las funciones nerviosas, que se acentúa en la vejez, trae como consecuencia, temblor y pérdida en la intensidad de la voz.

Según Garde (1973), en cuanto a la acción hormonal, ésta es determinante en la voz e Influye de manera importante en la excitabilidad del esfínter glótico. Con relación a los tejidos blandos, estos presentan un estado de edema crónico, puede observarse hipofunción velofaríngea y rinofonía.

El sistema respiratorio, presenta pérdida de la elasticidad de los pulmones, disminución de su volumen y de la elasticidad de la caja toráxica, atrofia de la musculatura respiratoria, que afecta, la ventilación pulmonar; la capacidad vital disminuye en un alto porcentaje. En este mismo trabajo se vio cómo la eficiencia respiratoria de un valor promedio de 15 segundos, en el adulto normal, a esta edad puede descender a 5 y 3 segundos, pudiéndose comprobar también una conservación significativa en personas que han sido profesionales de la voz, con una técnica vocal adecuada, como en el caso del cantante clásico.

Durante esta edad, también el tipo respiratorio se modifica, tendiendo a ser costal superior, en personas que no han tenido un manejo técnico de la voz. La postura también comienza afectarse, la columna cervical tiende a curvarse, se aumenta la xifosis dorsal y se inmobiliza la columna lumbar.

La mujer sufre un mayor deterioro de la voz, ésta se agrava dando timbres virilizados, según Linville (1996), en la mujer joven el tono fundamental es de $248 \mathrm{~Hz}$, y desciende a $175 \mathrm{~Hz}$ en la etapa senil suelen aparecer los temblores en la voz por la falta de control de la báscula laríngea y apoyo diafragmático, la respiración se altera y puede haber fatiga en el acto vocal.

En el hombre ocurre lo contrario, pues hay elevación de la frecuencia fundamental, según Linville (1996), el tono fundamental en el hombre joven está en una frecuencia de $110 \mathrm{~Hz}$ y sube a $148 \mathrm{~Hz}$ en el hombre a los noventa años.

Igualmente en esta etapa, la voz cantada no tiene justeza en la emisión y la reproducción de la melodía no es muy fiable, juega aquí un papel muy importante la audición, que en la mayoría de los casos evidencia una presbiacusia, que aparece por lo general, despues de los 70 años afectando la fineza de la discriminación auditiva, tanto para sonidos agudos como graves; este déficit auditivo hace perder el control auditivo fonatorio, trayendo como consecuencia, descenso de la frecuencia, pérdida de armónicos y alteraciones en la intensidad, de tal manera que la senilidad de la voz está influida por la senilidad auditiva, como afirma Augspach (1984) existe en la voz un deterioro biológico, fisiológico, orgánico y funcional que se va incrementando con la edad.

Perelló en 1980, resume así las características vocales de esta edad:

o Reducción de la extensión vocal

o El tono fundamental estaría en el hombre después de los 70 años entre los 125 y $130 \mathrm{~Hz}$., mientras que en la mujer desciende a $180 \mathrm{~Hz}$ y aún más bajo

o Aparece temblor en la voz

o Disminución de la intensidad

o Reducción de la resonancia

o Alteración de la coordinación fonorespiratoria, aumentándose la frecuencia respiratoria.

o Atrofia de los pliegues vocales

o Alteración del sistema endocrino

o Presbiacusia

o Alteración del sistema respiratorio

Se tiende a considerar que los cambios de la voz son inevitables, pero se ha podido comprobar, que estos pueden ser modificados con un trabajo vocal preventivo, que favorezca su conservación. Este trabajo fue propuesto por Aponte (1992), en la conferencia "conservación de la voz en la tercera edad", expuesto durante el seminario, "La voz Humana", realizado en la Escuela Colombiana de Rehabilitación, afiliada a la Universidad del Rosario, en la cual se exposo una intervención específica, sobre la musculatura que interviene en la fonación, respiración y voz, haciéndose especial énfasis en la voz cantada.

Según Pícoli (1984), se puede llevar a cabo una profilaxis preventiva para conservar la voz, pues la inercia lleva a la pérdida de funciones; estimulando la función, el órgano se puede conservar, porque la función hace al órgano.

Según Ávila, y otros autores (1993), en el trabajo sobre "La voz en la tercera y cuarta edad en profesionales y no profesionales de la voz", se pudo observar que en el cantante lírico, perteneciente a la cuarta edad, había una conservación del tono y timbre de la voz, sin presencia de temblor. 
Guerrero y otros autores (1994), afirman que la eficiencia respiratoria, la eficiencia respiratoria fonatoria y el tiempo máximo de fonación, se encuentran más conservados en los profesionales de la voz hablada y cantada, con y sin técnica vocal, que en aquellas personas que han hecho un uso normal de la voz y no han tenido ningún manejo técnico de la misma, observando un desempeño sobresaliente en el cantante lírico, de la cuarta edad, quien aún practicaba la técnica vocal.
Los modernos enfoques de la medicina actual, nos dan la pauta, para trabajar en pro de la conservación de la salud y en este caso de la salud vocal; no escatimar esfuerzos en la población de la tercera edad, que merece todo el apoyo. Así como desde la fonoaudiología, se brindan ayudas auditivas y de estimulación de lenguaje, sería conveniente trabajar con la voz, para retrazar la aparición de esta sintomatología, que inexorablemente se va a presentar, en la edad más avanzada. Brindar calidad de vida, es una de las metas de la rehabilitación.

\section{BIBLIOGRAFIA}

Aponte, $C$.: Conservación de la voz en la tercera edad, Seminario: La voz humana, Escuela Colombiana de Rehabilitación, afiliada a la Universidad del Rosario, 1992

Augspach, F.: El deterioro a lo largo de la vida de la voz, Rev. Fonoaudiológica, Buenos Aires,30,2, 1984 Avila, J., Barrios, F., Castillo, F., Correa, M., Peña, $N$.: La voz en la tercera y cuarta edad, en profesionales y no profesionales de la voz, Colegio Mayor de Nuestra Señora del Rosario, Seminario de voz y habla, Facultad de Fonoaudiología, Bogotá, 1993.

Dalleas, B.: Evolution de la voix de la naissaance a la puberté, Revue de Laryngologie, vol. 108, No 4, 1987 De Piccoli, E.: El deterioro vocal en la tercera edạd: Disfonías de involución, Rev. Fonoaudiológica, 30, 2 , 1984

Garde, E.: La voz, Editorial Central, Buenos Aires, 1973

Guerrero, A., Patiño, C., Pineda, M., Solarte, L.: La aerodinámica de la voz en personas de la cuarta edad profesionales de la voz cantada, hablada con y sin técnica vocal y en personas con uso normal de su voz, Escuela Colombiana de Rehabilitación, afiliada a la Universidad del Rosario, Seminario de voz y habla, Facultad de fonoaudiología, Bogotá, 1994

Heuillet, J.,Garson,Bavard, H., Legré, A.: Une voix pour tous, Tomo 2, Solal Editeurs, Marseille, 1996 Honjo, Iwao and Isshiki: Laryngoscopic and voice caracteristics of age persons, Arch Otolaryngol, vol 106, 1980

Linville,S.: The sound of Senescence, Journal of voice, Vol. 10, No 2, Philadelphia, 1996 Murray, M.,Rammage, L.: Tratamiento de los trastornos de la voz, Masson, S.A., Barcelona, 1996 Perelló, J.: Alteraciones de la voz, Editorial Científico Médica, Barcelona, 1980

Sataloff, $R$.: The effect of age on the voice, Professional voice care: the science and art of clinical care, Raven Press, Ltd., N.Y. 1991

Verhulst, J.: Evolution du larynx de la naissance a la puberté, Revue de Laryngologie, Vol. 108, No 4, 1987 Woisard, V., Percodani, J., Serrano, E., Pessey, J.J.: La voix de l'enfant, evolution morphologique du larynx et ses conséquences acoustiques, Revue de Laryngol, otol. Rhinol, 117, 1996 